\title{
Analytical solution of the steady Navier-Stokes equation for an incompressible fluid entrained by a rotating disk of finite radius using the apparatus of tunnel mathematics.
}

\author{
Shvydkyi O. G., Zaporozhe, Ukraine \\ e-mail: oleg.shv.jw@gmail.com
}

The flow in the neighborhood of a rotating disk is of great practical importance particularly in connexion with rotary machines. It becomes turbulent at larger Reynolds numbers, $R>3 \cdot 10^{5}$, in the same way as the flow about a plate. In this article we consider o motion of incompressible fluid that is always turbulent in circular direction (Reynolds number based on circular velocity $R_{\varphi}>3 \cdot 10^{5}$ ) and is of both of kinds in radial direction, i e. laminar (Reynolds number based on radial velocity $\left.R_{r}<3 \cdot 10^{5}\right)$ and turbulent $\left(R_{r}>3 \cdot 10^{5}\right)$. The equations of analyticity of functions of spatial complex variable (shortly, the equations of tunnel mathematics) affords a possibility to seek the solutions of steady Navier-Stokes equation in view of elementary functions. All vector fields, including those obeying the Navier-Stokes equation, satisfy to the equations of tunnel mathematics. The Navier-Stokes equations themselves are afterwards applied for verification of obtained solutions and calculation the pressure. Obtained formulae for pressure allow to visualize the presence of the boundary layer and estimate its thickness for laminar and turbulent flows. We use the Prandtl's concept of considering of fluids with small viscosities, i. e. we suppose that Reynolds number is enough large and the viscosity has in important effect on the motion of fluid only in a very small region near the disk (boundary layer). We also suppose that the fluid and the disk had at beginning the same temperatures and the energy dissipation occurs only by means internal friction.

\section{Introduction.}

The exact solution of the problem indicated in the title of this article for an infinite disk (the problem was stated by Karman) is given in the handbook on theoretical physics of Landau and Lifshitz [1] and in the handbook of Schlichting [3]. There this problem was reduced to solving a system of second-order ordinary differential equations which was obtained by numerical methods. The results of this solution are shown in Fig. 1.

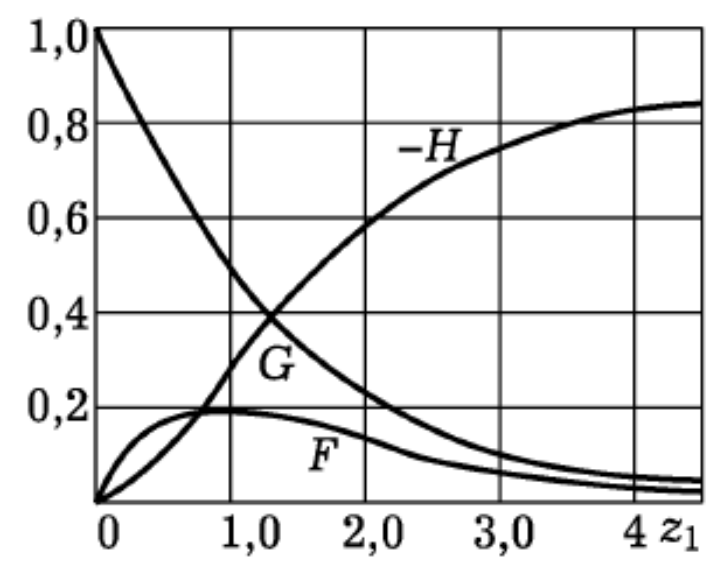

Fig. 1. Solution of the problem for an infinite disk using numerical methods in the handbook of Landau and Lifshitz.

In Fig. 1 the function $F$ corresponds to the radial velocity $v_{r}$, the function $G$ to the circular velocity $v_{\varphi}$ and the function $H$ to the axial projection $v_{z}$. 
In this case, the disk is located in the plane $z=0$ of cylindrical coordinates and rotates around the $z$ axis with a constant angular velocity $\Omega$. The liquid is considered from the side of the disk where $z>0$. The boundary conditions for the problem are as follows:

in the plane $z=0$ we have $v_{r}=0 ; v_{\varphi}=\Omega r ; v_{z}=0$,

i. e. the fluid adheres to the surface of disk. We assumed in our problems that radius of the disk is arbitrarily equal to unity. Although we have imposed no boundary conditions at $r=\infty$, and $z=H$, where $H$ is an immersion depth of the disk, the equation of tunnel mathematics allow to calculate correct solution of the problem at some distance from the edge of the disc (for $r$ approximately equal 2 ).

\section{Theory.}

This article provides a solution to a more realistic physical problem when a rotating disk has a finite radius (arbitrarily equal to unity). The solution applies only to the volume of the fluid enclosed in a cylinder of approximately unit radius of height $z_{l}$ (where $z_{l}$ is the height in limits of which the fluid moves in the horizontal mainly radial direction on Fig. 2).

As is known, if the nonlinear terms in the Navier-Stokes equations for a viscous fluid do not vanish identically then the solution of these equations presents great difficulties, and exact solutions can be obtained only in a very small number of cases. Concerning unsteady NavierStokes equation, T. Tao in his article [4] showed that it can have solutions which become infinite during finite time (blowup solutions). And if even finite solutions exist they are usually presented in the form of infinite power series what is inconvenient for use in practice. That's why it is reasonable to seek solutions of steady Navier-Stokes equation in the form of elementary functions. An exact solution to the problem under consideration (purely mathematical and also in the form of power series) was given by Miss D. M. Hannah [5], and A.N. Tifford [6] for the case of laminar flow; H. Schlichting and E. Truckenbrodt [7] provided an approximate solution with the aid of numerical methods. E. Truckenbrodt also investigated the case of turbulent flow. D. Weyburne [8] applied for describing of the boundary layer the standard probability distribution function methodology.

In this article the solution implies obtaining the analytical expressions (closed-form formulae, not power series) for three components of velocity and pressure in cylindrical polar coordinates. Numerical methods are not applicable. The formulae for the velocity components are derived from the relations of tunnel mathematics since latters are satisfied by all vector fields (including those obeying the Navier-Stokes equations). In this case unnecessary solutions are inevitably obtained and must be cut off by means of a physical analysis of the task. The Navier-Stokes equation is then used for verification of obtained solutions and calculation the pressure. Obtained formulae for pressure allow to visualize the presence of the boundary layer and estimate its thickness for laminar and turbulent flows.

Just as for an infinite disk the solution of the problem for a fluid entrained by a rotating disk of finite radius is symmetric with respect to the $\varphi$ coordinate (i. e. has axial symmetry). Fig. 2 schematically shows fluid streamlines in one of the planes parallel to the $z$ axis. 


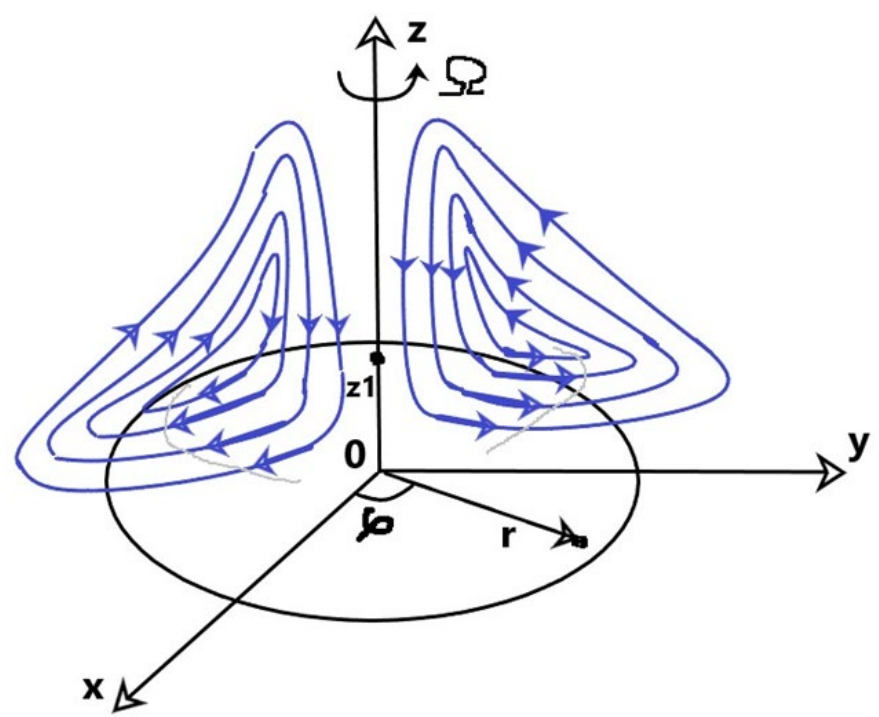

Fig. 2. Fluid streamlines in one of the planes parallel to the $z$ axis.

Tunnel mathematics equations applied to the components of the vector velocity field in Cartesian coordinate system look like this [9]:

$\frac{\partial u}{\partial x}+\frac{w x y}{\left(x^{2}+y^{2}\right)^{3 / 2}}=\frac{\partial v}{\partial y}-\frac{w x y}{\left(x^{2}+y^{2}\right)^{3 / 2}}=-\frac{2 y}{\sqrt{x^{2}+y^{2}}} \frac{\partial u}{\partial z}+\frac{\partial w}{\partial z}$.

$\frac{\partial v}{\partial x}+\frac{w y^{2}}{\left(x^{2}+y^{2}\right)^{3 / 2}}=-\frac{\partial u}{\partial y}+\frac{w x^{2}}{\left(x^{2}+y^{2}\right)^{3 / 2}}=-\frac{2 y}{\sqrt{x^{2}+y^{2}}} \frac{\partial v}{\partial z}$.

$\frac{\partial w}{\partial x}=-i \frac{\partial w}{\partial y}=-\frac{\partial u}{\partial z}-i \frac{\partial v}{\partial z}$

The components of the velocity field in expressions (2) - (4) are set as follows:

$V_{x}=u ; V_{y}=v ; V_{z}=R e w ;$

where $R e$ denotes real part of function; moreover, the functions $u, v, w$ are components of the function of the spatial complex variable [9]:

$P(L)=u(x, y, z)+i v(x, y, z)+f w(x, y, z)$

As usual the fluid is considered as incompressible:

$\nabla \cdot \boldsymbol{V}=\operatorname{div} \boldsymbol{V}=\frac{\partial u}{\partial x}+\frac{\partial v}{\partial y}+\frac{\partial w}{\partial z}=0$

Taking into account (7) we obtain from equations (2) such relation:

$\frac{\partial u}{\partial z}=0$

Relation (8) holds provided

$\frac{\partial w}{\partial z}=0$ 
We recall that function $w$ is an analytic function of the variables $x$ and $y$ and, therefore, a harmonic function on the $x y$ plane.

Any vector fields including those obeying the Navier-Stokes equations satisfy the relations (2) (4).

Taking into account the symmetry in the $\varphi$ coordinate the Navier-Stokes equations in cylindrical polar coordinates take the following form:

$\rho\left(v_{r} \frac{\partial v_{r}}{\partial r}+v_{z} \frac{\partial v_{r}}{\partial z}-\frac{v_{\varphi}^{2}}{r}\right)=-\frac{\partial P}{\partial r}+\mu\left(\frac{1}{r} \frac{\partial}{\partial r}\left(r \frac{\partial v_{r}}{\partial r}\right)+\frac{\partial^{2} v_{r}}{\partial z^{2}}-\frac{v_{r}}{r^{2}}\right) ;$

$\rho\left(v_{r} \frac{\partial v_{\varphi}}{\partial r}+v_{z} \frac{\partial v_{\varphi}}{\partial z}-\frac{v_{r} v_{\varphi}}{r}\right)=\mu\left(\frac{1}{r} \frac{\partial}{\partial r}\left(r \frac{\partial v_{\varphi}}{\partial r}\right)+\frac{\partial^{2} v_{\varphi}}{\partial z^{2}}-\frac{v_{\varphi}}{r^{2}}\right) ;$

$\rho\left(v_{r} \frac{\partial v_{z}}{\partial r}+v_{z} \frac{\partial v_{z}}{\partial z}\right)=-\rho g-\frac{\partial P}{\partial z}+\mu\left(\frac{1}{r} \frac{\partial}{\partial r}\left(r \frac{\partial v_{z}}{\partial r}\right)+\frac{\partial^{2} v_{z}}{\partial z^{2}}\right) ;$

where $g$ is the gravitational acceleration;

$P$ - pressure in fluid;

$\mu$ - coefficient of dynamic viscosity;

$\rho$ - fluid density.

Due to the fact that the Laplacian of the analytic function $w$ is equal to zero the equations of tunnel mathematics describe a fluid which moves along the $z$ axis without internal friction, i.e. as an ideal fluid (this is a feature of this method for incompressible fluid). Taking this into account we can obtain the following form of the function $w$ from equations (5), (9) and (12):

$w=\log e^{i \varphi}=\log r+i \varphi=\log \sqrt{x^{2}+y^{2}}+i \tan ^{-1} \frac{y}{x}$.

In order to comply with dimension, the expression (13) must be written in the following form:

$w=\Omega \mathrm{C}_{0}\left(\log \frac{r}{r_{0}}+i \varphi\right)=\Omega \mathrm{C}_{0}\left(\log \frac{\sqrt{x^{2}+y^{2}}}{r_{0}}+i \tan ^{-1} \frac{y}{x}\right)$

Where the constants have follow dimensions: $\left[C_{0}\right]=m,\left[r_{0}\right]=m$; we can impose $r_{0}=1 \mathrm{~m}$. Constant $r o$ allows to adjust the arbitrary radius of the disk.

We find such relations after differentiating (14):

$\frac{\partial w}{\partial x}=\frac{\Omega \mathrm{C}_{0}(x-i y)}{x^{2}+y^{2}} ;$

$\frac{\partial w}{\partial y}=\frac{\partial v}{\partial z}=\frac{\Omega \mathrm{C}_{0}(y+i x)}{x^{2}+y^{2}}=\frac{\Omega \mathrm{C}_{0} f^{*}}{\sqrt{x^{2}+y^{2}}}=\frac{\Omega \mathrm{C}_{0}\left(f+\frac{2 y}{\sqrt{x^{2}+y^{2}}}\right)}{\sqrt{x^{2}+y^{2}}}=\frac{\Omega \mathrm{C}_{0}(f+2 \sin \varphi)}{r}$.

Expression (16) uses the following relations of tunnel mathematics for the operator $f$ and its conjugate operator $f^{*}[9]$ :

$$
\begin{aligned}
& f=i e^{i \varphi}=-\sin \varphi+i \cos \varphi=\frac{-y+i x}{\sqrt{x^{2}+y^{2}}} \\
& f^{*}=i e^{-i \varphi}=\sin \varphi+i \cos \varphi=\frac{y+i x}{\sqrt{x^{2}+y^{2}}}
\end{aligned}
$$


$f \times f^{*}=-1$.

$f^{*}=f+2 \sin \varphi$.

Special attention should be paid to the operator $f$ in (16) since the factor at it will be used to reconstruct the spatial coordinate $z$ in the equations on the plane (what corresponds to the relation (6)). We can see from equation (16) that this factor is $\frac{1}{r}$ (we omit constants and term depending on $\varphi$ ).

Generally speaking, the complex function $w$ must be selected in such a way that its real part to be corresponding to the pattern of occurring physical phenomena (i. e. to the projection $V_{z}$ of the velocity field in Fig. 2), and the integrating of relations (2) and (3) does not generate integrals that could not be solved in quadratures.

The form of the real part of the function $w$ according to expression (14) is shown in Fig. 3.

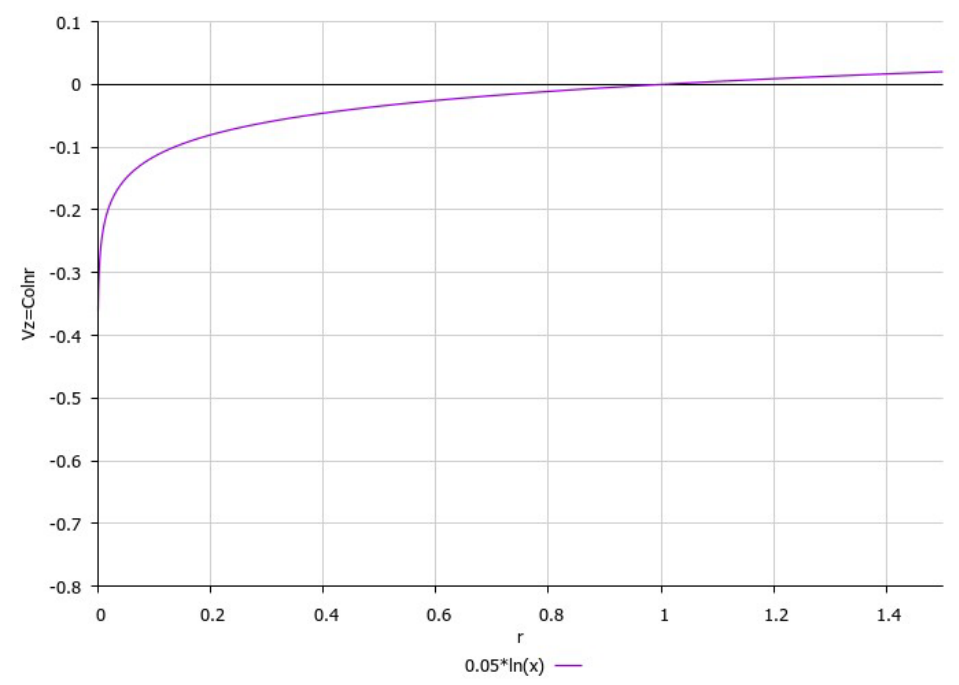

Fig. 3. The graph of the real part of the function $w\left(C_{0}=0.05 \mathrm{~m}\right)$.

It can be seen from the graph shown in Fig. 3 that the radius of the rotating disk should be equal to unity (arbitrarily). For $r>1$ the fluid streamlines are already rising.

This form of the real part of the function $w$ is inapplicable in the vicinity of the point $r=0$ since the graph goes to infinity.

Transforming relations (2) and (3) to cylindrical polar coordinates and taking into account expressions (8) and (9) we obtain:

$\frac{\partial v_{r}}{\partial r}-\frac{\partial v_{\varphi}}{\partial r} \tan \varphi+\frac{v_{r}}{r}+\frac{v_{\varphi}}{r} \cot \varphi+\frac{w \sin \varphi \cos \varphi}{r}$
$=\frac{\partial v_{r}}{\partial r}+\frac{\partial v_{\varphi}}{\partial r} \cot \varphi+\frac{v_{r}}{r}-\frac{v_{\varphi}}{r} \tan \varphi-\frac{w \sin \varphi \cos \varphi}{r}=0 ;$

$\frac{\partial v_{r}}{\partial r} \tan \varphi+\frac{\partial v_{\varphi}}{\partial r}-\frac{v_{r}}{r} \cot \varphi+\frac{v_{\varphi}}{r}+\frac{w \sin ^{2} \varphi}{r}$

$=-\frac{\partial v_{r}}{\partial r} \cot \varphi+\frac{\partial v_{\varphi}}{\partial r}+\frac{v_{r}}{r} \tan \varphi+\frac{v_{\varphi}}{r}+\frac{w \cos ^{2} \varphi}{r}=-\frac{(2 \sin \varphi) \Omega \mathrm{C}_{0}(f+2 \sin \varphi)}{r}$. 
When deriving equations (21) and (22) the well-known formulas were used for the transition from Cartesian velocity projections to cylindrical polar ones:

$u=v_{r} \cos \varphi-v_{\varphi} \sin \varphi$;

$v=v_{r} \sin \varphi+v_{\varphi} \cos \varphi$.

Since in the region under consideration (Fig. 2) the liquid moves horizontally (mainly radially), when solving equations (21) and (22) we can put $w=0$. By integrating these equations under such condition we obtain two solutions for each of the projections of the velocity $v_{r}$ и $v_{\varphi}$. The solutions that meet to the physical pattern of the phenomena of this problem (particularly the boundary conditions (1)) are as follows:

$v_{\varphi}=\Omega r$

$v_{r}=\frac{\Omega \sigma}{r}$

where $\sigma$ is a constant of integration.

Expressions (25) and (26) are the solutions of equations (21).

Expression (26) holds since the solution should not have a dependence on $\varphi$, and we work with the assumption (9). Expression (26) is true for $r>1$, however, it doesn 't work for $r \rightarrow 0$ (it tends to infinity). That's why it need a correction.

\section{Results and discussions.}

To extend solutions (25) and (26) into space we use the factor at the operator $f$ in equation (22):

$z=\frac{1}{r}$

We consider $\sin \varphi$ at (22) as a constant.

The specific form of the velocity projection $v_{r}$ depends on the integral relation representing the corresponding mass fluxes of fluid through the surfaces of the cylinder covering the region in question (Fig. 2).

\subsection{Extension of solution on a plane into space for radial velocity.}

So, for the projection $v_{r}$ the following integral relation is used:

$\int v_{z} r d r=\int v_{r} d z$

where the integral on the left-hand side represents the mass flux entering through the upper end of the cylinder and the integral on the right-hand side represents the mass flux that is scattered through the side surface of the cylinder.

Taking into account equalities (5), (14) and (26), we can rewrite the integral relation (28) in following manner:

$$
\int r \log r d r=\int \frac{1}{r} d z
$$

where constants are omitted. 
Accordingly (27), the presence of the factor $\frac{1}{r}$ in the right-hand side of (29) is sufficient for building of spatial formula. In order to the integral in the right-hand side of (29) doesn't depend of changing the variable of integration $z$ by $r$, both variables $r$ and $z$ should enter in the expression for radial velocity $v_{r}$ symmetrically. Then formula (26) can be extended into space as follows:

$v_{r}=-\Omega \frac{r z}{z_{0 r}} \log \frac{r z}{r_{0} z_{0 r}}$

where the range of $r$ is about $(0 ; 2)$; the range of $z$ is $\left(0 ; z_{1}\right]$.

In (30) $z_{0 r}$ is a normalization factor, its dimension is meter. The value of $z_{0 r}$ depends from value of $z$ coordinate at which the Navier-Stokes equation works. Particularly, for $z$ coordinate corresponding to the boundary layer the value of $z_{0 r}$ is of the order of the thickness of boundary layer $\delta$, i. e. $z_{0 r}=\delta$.

Approximate spatial and plane graphs for the functions $v_{r}$ in (30) and corrected (26) are shown in Fig. 4 and 5 respectively.

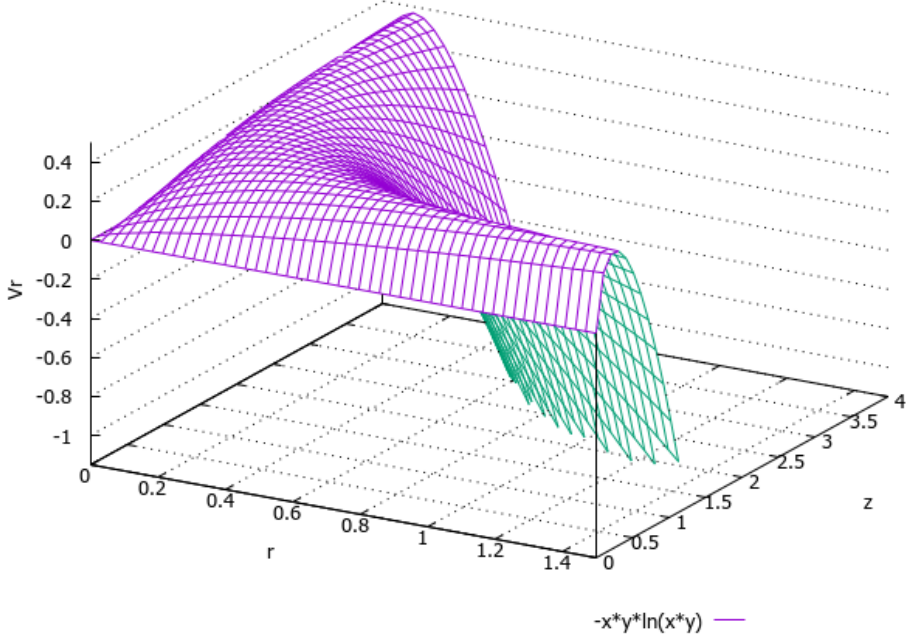

Fig. 4. Spatial graph for formula (30).

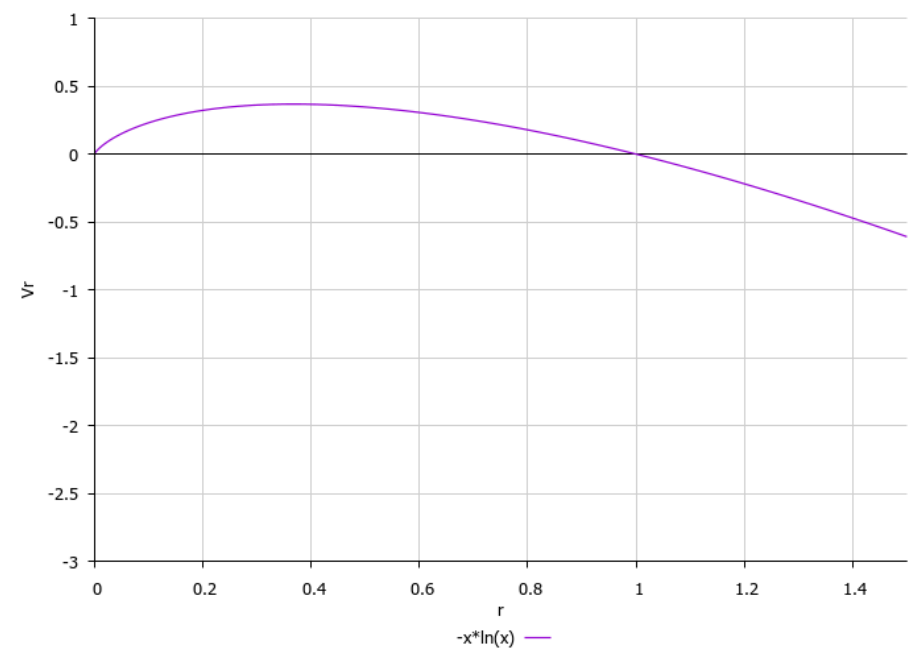

Fig. 5. Plane graph for corrected formula (26). 
These graphs correspond to the pattern of physical phenomena arising during the rotation of a disk in a liquid for the following reasons. Let us consider from above the motion of a liquid over a disk at rest in a non-inertial frame of reference for a small value of $z$ coordinate, when the cohesion forces of liquid particles with the disk surface still have an appreciable value (Fig. 6).

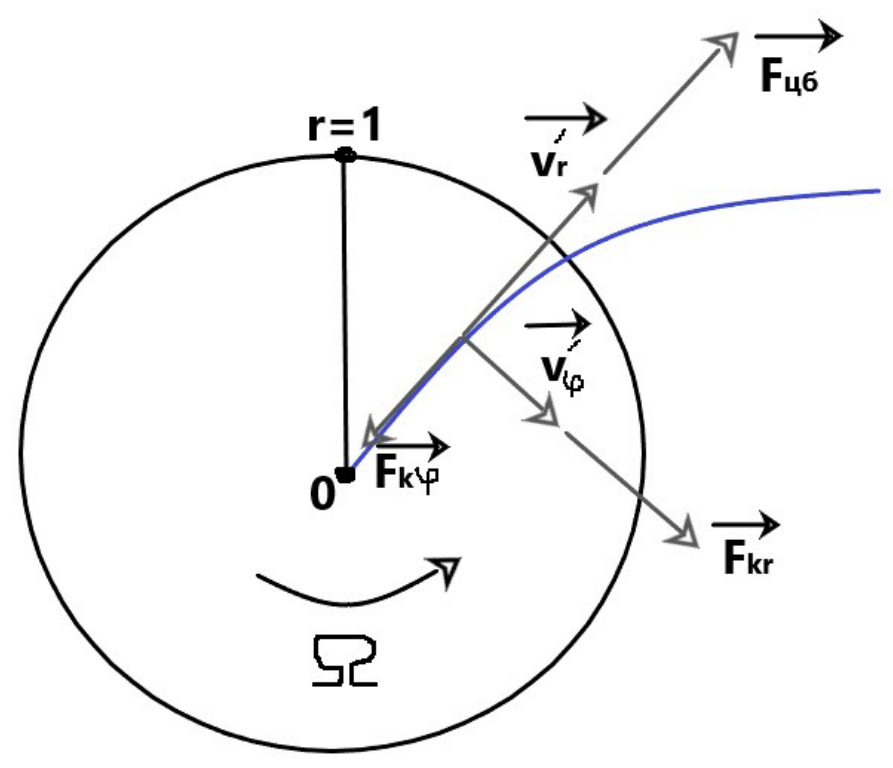

Fig. 6. Motion of liquid over a disk at rest in a non-inertial frame of reference for a small value of $z$ coordinate.

Coriolis forces $\overrightarrow{F_{k \varphi}}$ due to the circular velocity $\overrightarrow{v_{\varphi}^{\prime}}$ (the prime means movement in a non-inertial frame of reference) will be directed to the origin and thus will prevent the increase in radial

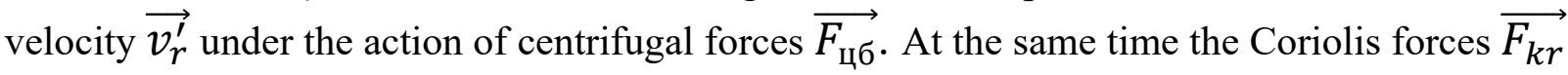
due to the radial velocity $\overrightarrow{v_{r}^{\prime}}$ will contribute to an increase in magnitude of the circular velocity $\overrightarrow{v_{\varphi}^{\prime}}$. As a result of such a dynamic pattern the radial velocity $\overrightarrow{v_{r}^{\prime}}$ having reached its maximum will gradually decrease to zero what corresponds to the graphs shown in Fig. 4 and 5.

A similar picture for the same reasons will be observed as $z$ coordinate increases while the $r$ coordinate remains constant. In this case the radial velocity $\overrightarrow{v_{r}^{\prime}}$ begins to increase due to the weakening of the cohesion forces between the liquid particles and the disk surface.

This dependence of the radial velocity component $v_{r}$ on the coordinates $r$ and $z$ can also be explained by energy considerations. If we return to the inertial reference system, then the appearance of a new component of velocity (radial) in it requires certain energy expenditures.

Since the energy supplied to the disk for its rotation remains unchanged, an increase in the radial component of the velocity $v_{r}$ is possible only due to a decrease in the circular component $v_{\varphi}$ which in the inertial frame of reference will tend to retain its value. Therefore, the process of extinguishing the radial component $v_{r}$ in both directions, $r$ and $z$, will inevitably occur in the liquid.

In this regard a natural question arises as to why in the handbook of Landau and Lifshitz [1] and in the handbook of Schlichting [3] the radial velocity depends on the coordinate $r$ according to linear law:

$v_{r}=r \Omega F(z)$. 
The point is that formula (31) really takes place for small values of the coordinate $r$. And since for an infinite disk (namely such a model is considered in the handbook of Landau and Lifshitz and in the handbook of Schlichting) any value of $r$ in principle can be considered as small, then formula (31) has a full right to live. It is of course inapplicable for a disk of finite radius.

Since in the region under consideration in Fig. 2 the liquid moves horizontally (mainly radially), then the projection of the particles velocity on the $z$ axis is zero $\left(v_{z}=0\right)$, and therefore Liouville's theorem on the invariability of the phase volume of a system of particles is satisfied automatically [2]. The fivefold integral $\int d r d z d v_{r} d v_{\varphi} d v_{z}$ is identically equal to zero.

It should also be noted that in accordance with the graphs in Figs. 4 and 5 for $r>1$ the fluid streamlines must abruptly change the direction of motion so that the vector of the total velocity makes an obtuse angle with the $r$ axis as actually shown in Fig. 2.

\subsection{Extension of solution on a plane into space for circular velocity.}

By means (27) the extension of (25) into space is fulfilled very simple.

$v_{\varphi}=\frac{\Omega r}{\frac{z}{z_{0 \varphi}}+1}$

where $Z_{0 \varphi}$ is a normalization factor, its dimension is meter. We added unity in denominator in order to satisfy the boundary conditions (1).

\subsection{Finding the auxiliary relations.}

We can at once verify the solutions (30) and (32) that have been expanded into space by means apparatus of tunnel mathematics with the aid of true Navier-Stokes equations. Simplest way to do this is to use equation (11) since it doesn't contain the pressure $P$. Substituting solutions (30) and (32), and real part of relation (14), and their corresponding derivative into equation (11) we conclude following important auxiliary relation for $z$ coordinate:

$Z+z_{0 \varphi}=-\frac{2 \mu}{\rho \Omega \mathrm{C}_{0} \log \frac{r}{r_{0}}}$

If $z_{0 \varphi}$ is small then

$Z=-\frac{2 \mu}{\rho \Omega \mathrm{C}_{0} \log \frac{r}{r_{0}}}$

Relation (34) connects the important quantities for fluid, such as coefficient of dynamic viscosity $\mu$, fluid density $\rho$, and angular velocity $\Omega$. Adjusting angular velocity $\Omega$ (what corresponds to adjusting of Reynolds number) we can regulate magnitude of $z$ coordinate on which the NavierStokes equations work.

We can conclude from (34) that inequality $r_{0}>r$ must hold. Solving our problem, we put $r_{0}=1$. 
Formula (11) where the pressure is absent due to axial symmetry can be used to calculate the friction force acting in a fluid including that acting per unit disk surface in a direction perpendicular to its radius. Having performed the appropriate calculations with (32), we obtain the following formula for the friction force:

$$
F_{f r}=\mu \frac{\partial v_{\varphi}}{\partial z}=-\frac{\mu \Omega r}{z_{0 \varphi}\left(\frac{z}{z_{0 \varphi}}+1\right)^{2}} .
$$

Directing $z$ coordinate to zero in expression (35) we obtain the relation for the friction force acting on a unit surface of the disk in the direction perpendicular to its radius:

$F_{f r}=-\frac{\mu \Omega r}{z_{0 \varphi}}$

Thus we conclude from (36) that constant $z_{0 \varphi}$ for laminar and turbulent motion of fluid must be determined experimentally.

\subsection{Calculation of pressure and parameters of the boundary layer using the Navier-Stokes equations.}

Using this method, it is still quite difficult to obtain an exact analytical expression for pressure since, for example, equation (12) for projections of velocity on $z$ axis works only for an ideal fluid (i. e. this equation doesn`t work fully in the region of the boundary layer).

The approximate formula for the boundary layer in the area under consideration in Fig. 2 looks like this:

$$
\begin{gathered}
\left.P=-\frac{\rho(\Omega r)^{2}}{2}\left(\frac{z}{\delta} \log \frac{r z}{r_{0} \delta}\right)^{2}-\frac{C_{0}}{\delta}\left(\log ^{2} \frac{r}{r_{0}}+\left(\log \frac{r}{r_{0}}-\frac{1}{2}\right) \log \frac{z}{\delta}\right)-\frac{1}{\left(\frac{z}{z_{0 \varphi}}+1\right)^{2}}\right) \\
-\frac{\mu \Omega}{2 \delta}\left(4 z \log \frac{r z}{r_{0} \delta}+\frac{r^{2}}{z}\right) .
\end{gathered}
$$

Formula (37) is obtained by integrating of equation (10). Since (37) referred to the region of boundary layer we replaced the constant $z_{0 r}$ by the thickness of boundary layer $\delta$.

In this case the corresponding derivatives were calculated using formulae (14), (30) and (32). Generally speaking, we must yet add to right-hand side of (37) the hydrostatic pressure $\rho g(H-z)$, where $H$ is an immersion depth of the disk.

In Fig. 7 and 8 graphs corresponding to formula (37) in the region of laminar boundary layer (Reynolds number based on radial velocity $R_{r}=\frac{v_{r} r}{v} \approx \frac{\Omega z r}{v}<3 \cdot 10^{5}$, where $v$ is a kinematic viscosity of fluid) are presented at values of angular velocity $\Omega$ equal $70 \mathrm{rad} / \mathrm{s}$ and $100 \mathrm{rad} / \mathrm{s}$ respectively with using the following values of the constants:

$$
\begin{aligned}
& z_{0 \varphi}=0.001 \mathrm{~m} ; \\
& \delta=z_{0 r}=0.00025 \mathrm{~m} ; \\
& C_{0}=0.001 \mathrm{~m} ; \\
& r_{0}=1 \mathrm{~m} .
\end{aligned}
$$


On all figures shown below the pressure is measured in Pascals, and $r$ and $z$ coordinates are measured in meters.

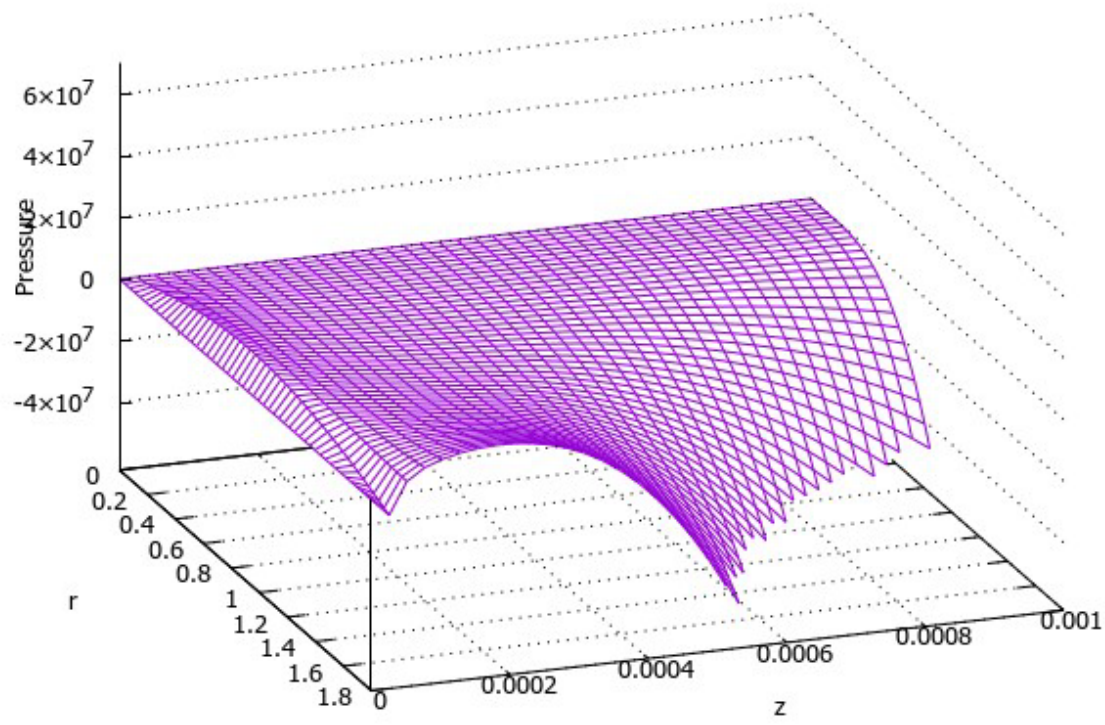

$\left./ 0.00025)) *(\ln (x / 1)-0.5))-1 /(y / 0.001+1)^{2}\right)-(0.001 * 70 /(2 * 0.00025)) *\left(4 * y^{*} \ln \left(x^{*} y /(1 * 0.00025)\right)+x^{2} / y\right)-$

Fig. 7. Graph corresponding to formula (37) at $\Omega=70 \mathrm{rad} / \mathrm{s}$ (laminar motion in the radial direction).

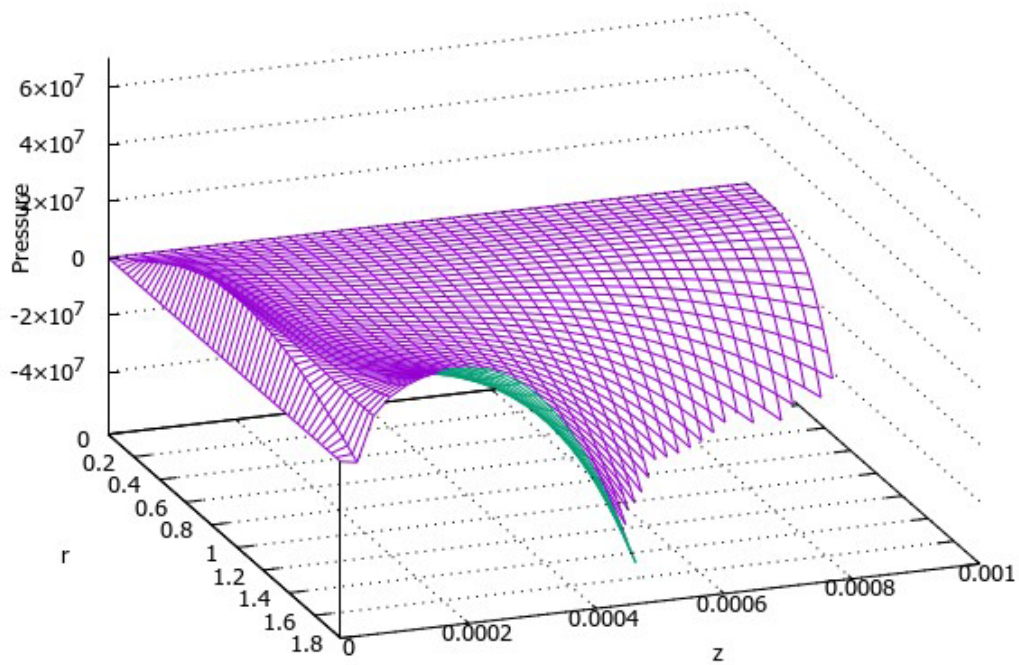

$\left.0.00025)) *(\ln (x / 1)-0.5))-1 /(y / 0.001+1)^{2}\right)-(0.001 * 100 /(2 * 0.00025)) *\left(4^{*} y^{*} \ln \left(x^{*} y /(1 * 0.00025)\right)+x^{2} / y\right)-$

Fig. 8. Graph corresponding to formula (37) at $\Omega=100 \mathrm{rad} / \mathrm{s}$ (laminar motion in the radial direction).

We recall that radius of disc is equal to 1 meter. 
The graphs are calculated for water with a density $\rho=1000 \mathrm{~kg} / \mathrm{m}^{3}$ and a dynamic viscosity coefficient $\mu=0.001 \mathrm{~Pa} \times_{S}$. We can see from these graphs that the pressure profile in boundary layer of incompressible fluid for our problem is convex. In the thin boundary layer (very small $z$ ) the pressure significantly arises what corresponds to the presence of a large tangential-velocity gradient, resulting in a large viscous dissipation of energy [1].

We conclude from (38) that the thickness of laminar boundary layer is of the order $\delta \sim 2.5 \cdot 10^{-4} \mathrm{~m}$, what fully corresponds to the theory of incompressible fluid [1], [3]:

$\delta \sim \sqrt{\frac{v}{\Omega}}=\sqrt{\frac{10^{-6} \mathrm{~m}^{2} / \mathrm{s}}{1001 / \mathrm{s}}}=10^{-4} \mathrm{~m}$

In Fig. 9 graph corresponding to formula (37) in the region of turbulent boundary layer (Reynolds number based on radial velocity $R_{r}=\frac{v_{r} r}{v} \approx \frac{\Omega z r}{v} \sim 10^{8}$ ) are presented at value of angular velocity $\Omega$ equal $10000 \mathrm{rad} / \mathrm{s}$ with using the following values of the constants:

$z_{0 \varphi}=0.0001 \mathrm{~m}$

$\delta=z_{0 r}=0.025 \mathrm{~m}$;

$C_{0}=0.001 \mathrm{~m}$;

$r_{0}=1 \mathrm{~m}$.

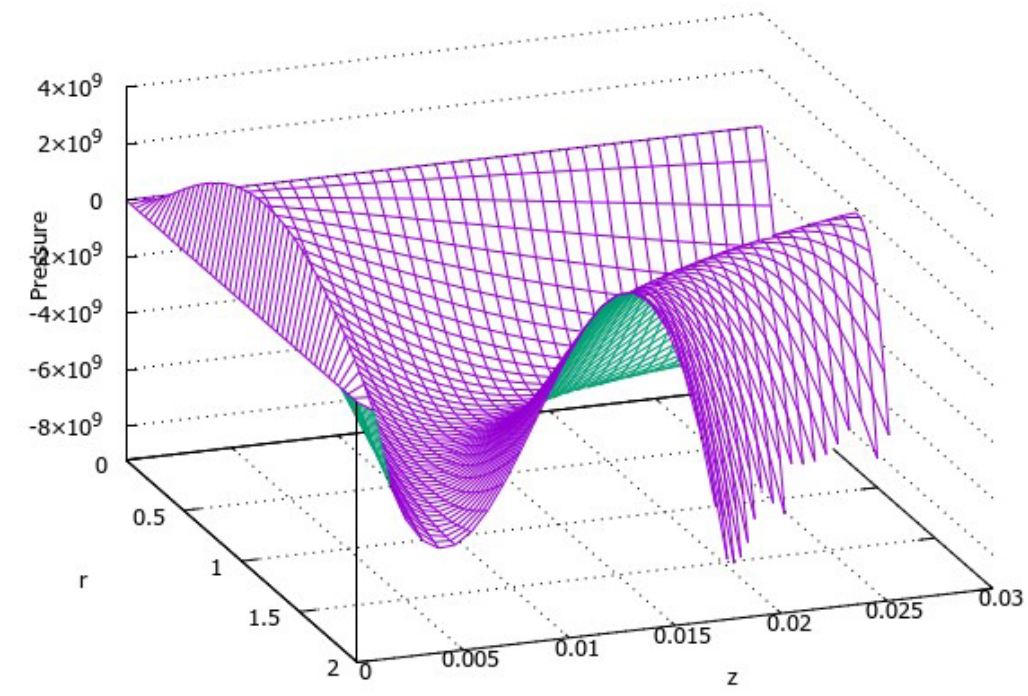

$\left.((y / 0.025)) *(\ln (x / 1)-0.5))-1 /(y / 0.0001+1)^{2}\right)-(0.001 * 10000 /(2 * 0.025))^{*}\left(4^{*} y^{*} \ln \left(x^{*} y /\left(1^{*} 0.025\right)\right)+x^{2} / y\right)$

Fig. 9. Graph corresponding to formula (37) at $\Omega=10000 \mathrm{rad} / \mathrm{s}$ (turbulent motion in the radial direction).

We conclude from (40) that the thickness of turbulent boundary layer is of the order $\delta \sim 2.5 \cdot 10^{-2} \mathrm{~m}$, what fully corresponds to the theory of incompressible fluid [3]:

$\delta \sim r^{3 / 5}\left(\frac{v}{\Omega}\right)^{1 / 5}=r^{3 / 5}\left(\frac{10^{-6} m^{2} / s}{100001 / s}\right)^{1 / 5}=r^{3 / 5} \cdot 10^{-2} \mathrm{~m}$ 
Now we verify either one can to find the relations corresponding to (39) and (41) of the theory of boundary layer from obtained formulae for pressure.

Integrating (12) we obtain following expression for pressure in the region of the boundary layer:

$$
P=-\frac{\rho\left(\Omega C_{0}\right)^{2}}{2 \delta}\left(-\frac{z^{2}}{C_{0}}\left(\log \frac{r z}{r_{0} \delta}-\frac{1}{2}\right)+\delta \log ^{2} \frac{r}{r_{0}}+\frac{2 \delta}{\left(\Omega C_{0}\right)^{2}} g z\right) .
$$

Corresponding derivatives were calculated using formulae (14) and (30). We omit the constants of integration. In (42) the term with $\mu$ is absent.

Comparing (37) and (42) we find:

$$
\begin{gathered}
r^{2}\left(\left(\frac{z}{\delta} \log \frac{r z}{r_{0} \delta}\right)^{2}-\frac{C_{0}}{\delta}\left(\log \frac{r}{r_{0}}+\left(\log \frac{r}{r_{0}}-\frac{1}{2}\right) \log \frac{z}{\delta}\right)-\frac{1}{\left(\frac{z}{z_{0 \varphi}}+1\right)^{2}}\right) \\
=-\frac{z^{2} C_{0}}{\delta}\left(\log \frac{r z}{r_{0} \delta}-\frac{1}{2}\right)+C_{0}^{2} \log ^{2} \frac{r}{r_{0}}-\frac{2 g H}{\Omega^{2}}
\end{gathered}
$$

where $H$ is an immersion depth of the disk.

We can neglect in (43) the terms with $C_{0}$ as we can arbitrarily take its value very small. Besides, we consider polar coordinate $r$ as a constant.

Such ratios in the region of boundary layer follow from values of constants in (38) and (40):

$$
\frac{z}{\delta} \sim 1
$$

for laminar layer: $\frac{z}{z_{0 \varphi}} \sim 0$;

for turbulent layer: $\frac{z}{z_{0 \varphi}} \gg 1$.

Expanding the logarithms in (43) in Taylor's series near 1 as far as linear terms and neglecting infinitesimal terms we obtain following approximate equalities.

For laminar layer:

$r^{2}\left(\left(\frac{z}{\delta}\left(\frac{z}{\delta}-1\right)\right)^{2}-1\right)=-\frac{2 g H}{\Omega^{2}}$.

It's easy to see from (47) that maximum power of $z$ coordinate is 4 ; therefore, following relation at the same $r$ coordinate holds:

$Z \sim \frac{\text { const }}{\sqrt{\Omega}}$

It follows from (48) that the thickness of laminar boundary layer decreases inversely proportionally to square root of $\Omega$. This fully corresponds to (39) and the theory of laminar boundary layer in incompressible fluid [1], [3]. 
For turbulent layer:

$r^{2}\left(\left(\frac{z}{\delta}\left(\frac{z}{\delta}-1\right)\right)^{2}-\left(\frac{z_{0 \varphi}}{z}\right)^{2}\right)=-\frac{2 g H}{\Omega^{2}}$.

We can rewrite (49) in such matter:

$$
\left(\frac{z^{2}}{\delta z_{0 \varphi}}\left(\frac{z}{\delta}-1\right)\right)^{2}+2 g H\left(\frac{z}{\Omega r z_{0 \varphi}}\right)^{2}-1=0 .
$$

To find exact solutions of (50) is difficultly enough. But we know accordingly (41) that $z$ coordinate in the region of turbulent boundary layer must be of order of $0.01 \mathrm{~m}$. So, we must determine either roots of such order exist for equation (50).

In (50) we have:

$\Omega=10000 \mathrm{rad} / \mathrm{s}$

$z_{0 \varphi} \sim 0.0001 m ; \delta \sim 0.025 m$ (accordingly with (40));

$g=10 \mathrm{~m} / \mathrm{s}^{2}$

besides, we put $r \sim 0.5 \mathrm{~m}$.

In Figs. 10, 11, 12 the roots of (50) are shown versus an immersion depth of the disk $H$.

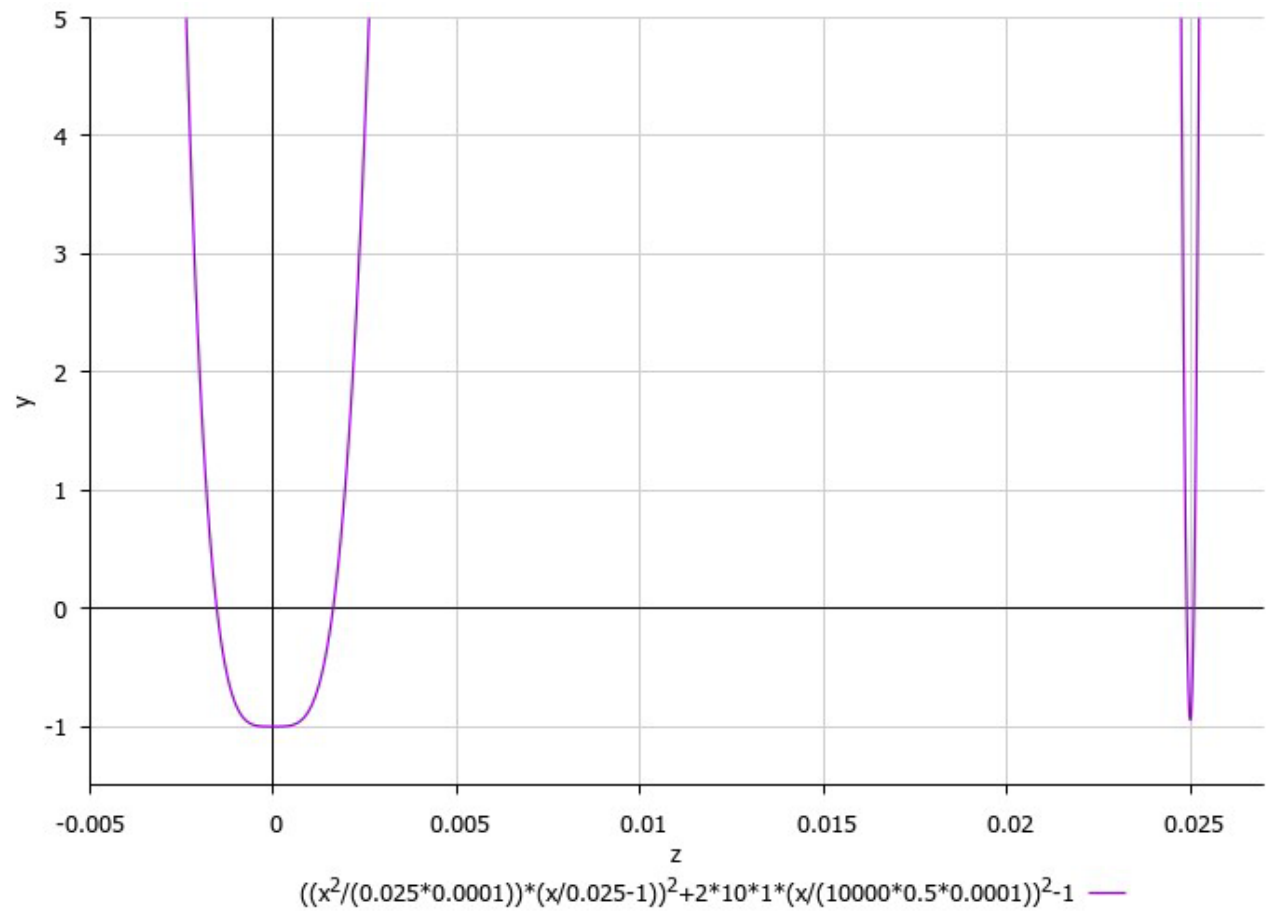

Fig. 10. Roots of equation (50) at $H=1 \mathrm{~m}$. 


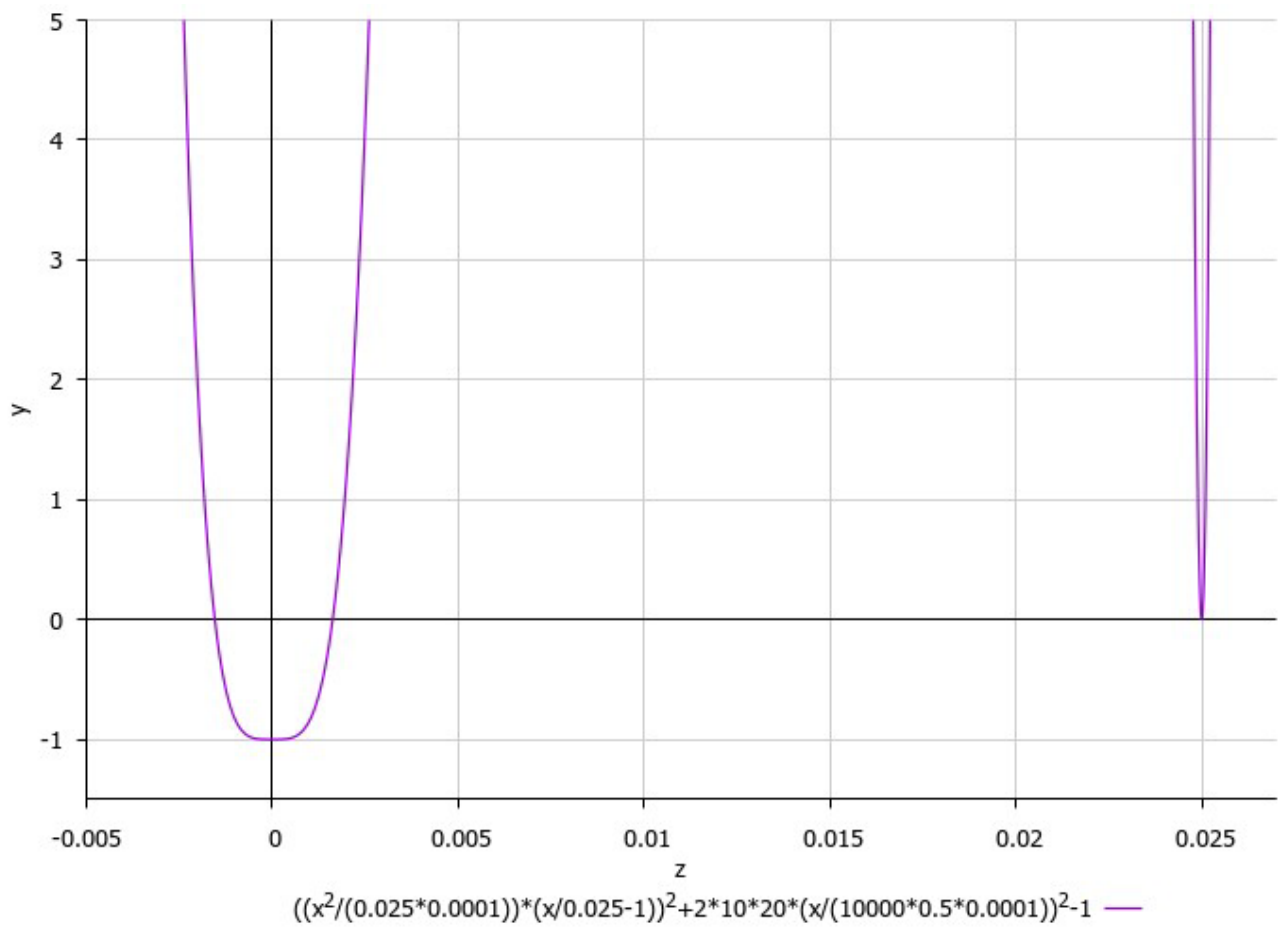

Fig. 11. Roots of equation (50) at $H=20 \mathrm{~m}$.

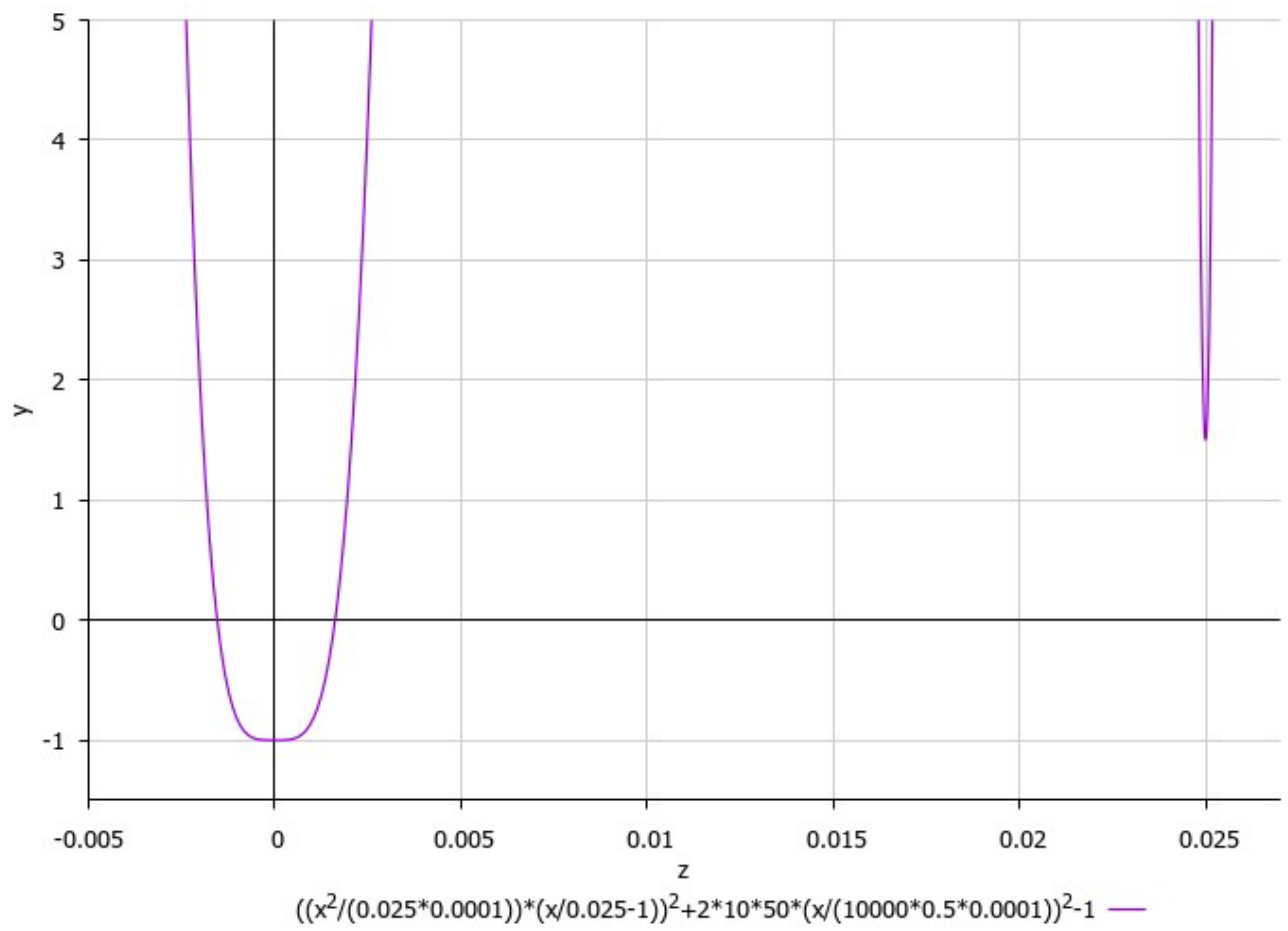

Fig. 12. Roots of equation (50) at $H=50 \mathrm{~m}$.

So, we see that at $H=1 \mathrm{~m}$ there are two appropriate roots near $z=0.025 \mathrm{~m}$; at $H=20 \mathrm{~m}$ there is one appropriate root equal exactly $0.025 \mathrm{~m}$; and at $H=50 \mathrm{~m}$ there are already no appropriate roots. Therefore, adjusting the magnitude of $H$ we can always find solutions of (50) in the region of turbulent layer. 


\section{Conclusions}

The solution of the Navier-Stokes equations using the tunnel mathematics apparatus is simple and elegant, and also requires a good mathematical training and a deep physical analysis of the problem. This method does not require a specific software and can be used for the primary analysis of hydrodynamic problems. Obtained formulae for pressure allow to visualize the presence of the boundary layer and estimate the order of its thickness for laminar and turbulent flows. The results obtained using this method for a fluid entrained by a disk of finite radius correspond to the results of a similar problem in classic handbooks on hydrodynamics where numerical methods were used.

\section{Reference list}

[1] Landau and Lifshitz (1987). "Fluid Mechanics," Course of Theoretical Physics, Volume 6, pp. 75-77, 162, 315.

[2] Landau and Lifshitz (1982). "Mechanics," Course of Theoretical Physics, Volume 1, pp. 146-148.

[3] Schlichting, Hermann (1979). "Boundary-Layer Theory," 7th ed., McGraw Hill, New York, U.S.A, pp. 102-106, 647, 648.

[4] Tao T., arXiv: 1402.0290v3 [math.AP] 1 Apr. 2015, Finite time blowup for an averaged three-dimensional Navier-Stokes equation.

[5] Hannah D. M. (1952). "Forced flow against a rotating disc," ARC/R\&M-2772.

[6] Tifford A. N. and Chu S. T. (1952). "On the Flow Around a Rotating Disc in a Uniform Stream," JAS 19, $284-285$.

[7] Schlichting H. and Truckenbrodt E. (1951). "The Flow Around a Rotating Disc in a Uniform Stream,” Readers 'Forum. Journal of the Aeronautical Sciences, Vol. 18 Num. 9, pp. $639-640$.

[8] Weyburne, David (2006). "A mathematical description of the fluid boundary layer," Applied Mathematics and Computation, vol. 175, pp. 1675-1684.

[9] Shvydkyi O. G. (2022). Application of tunnel mathematics for solving the steady

Lamé and Navier-Stokes equations, DOI:10.21203/rs.3.rs-1327681/v1

\section{6. 'Declaration of Interests. The authors report no conflict of interest.'}

\section{Data availability}

The data that supports the findings of this study are available within the article. 